\section{Evaluación de la Respuesta Superovulatoria en vaquillas Simmental del núcleo genético de la Universidad Nacional Tori- bio Rodríguez de Mendoza, Amazonas}

\section{Evaluation of the Superovulatory Response in heifers Simmental of the genetic nucleus of the National University Toribio Rodríguez de Mendoza, Amazonas}

Luis Murga', Jhony Gonzales ${ }^{2}$ y William Bardales ${ }^{3}$

\section{RESUMER}

El objetivo del presente estudio fue evaluar la eficiencia de un protocolo de superovulación en vaquillas de raza Simmental de la Universidad Nacional Toribio Rodríguez de Mendoza. Se realizaron 58 superovulaciones a once vaquillas en base al protocolo reportado por Bó et al. (2006). Se colectaron embriones con el método no quirúrgico y se clasificaron de acuerdo a la IETS (Sociedad Internacional de Transferencia de embriones). El número total de embriones obtenido fue 247 de los cuales 80,16 \% fueron mórulas; el 12,55 $\%$, blastocistos temprano; el 5,67 \%, blastocistos y el 1,62 \%, blastocistos expandidos. Se obtuvo, en promedio, 7,18 \pm 4,33 estructuras, 4,26 \pm 3,56 embriones transferibles y 1,76 $\pm 1,99$ embriones degenerados. Se encontró diferencia estadística, según el número de superovulaciones sucesivas sobre la producción de estructuras y embriones transferibles. En todos los criterios de evaluación del presente estudio, la respuesta superovulatoria respecto al número de estructuras y de embriones transferibles fueron las variables que se vieron significativamente afectadas. Esto permite concluir que las vaquillas de raza Simmental tuvieron una respuesta superovulatoria óptima al protocolo aplicado.

\section{PhLABRAS Clave}

Ovocitos $\cdot$ estadíos $\cdot$ donadoras

\section{ABSTRACT}

The objective of the present study was to evaluate the efficiency of a superovulation protocol in Simmental heifers of the National University Toribio Rodríguez de Mendoza. Eleven heifers were used, and 58 superovulations were performed based on the protocol reported by Bo et al., 2006. The embryos were collected using the non-surgical method and classified according to IETS Of Embryo Transfer). The total number of embryos obtained was 247 of which $80.16 \%$ were morulae, $12.55 \%$ were early blastocysts, $5.67 \%$ were blastocysts and $1.62 \%$ were expanded blastocysts. An average of $7.18 \pm 4.33$ structures, $4.26 \pm 3.56$ transferable embryos and $1.76 \pm 1.99$ degenerate embryos. Statistical difference was found, according to the number of successive superovulations on the production of transferable structures and embryos. In all the evaluation criteria of the present study the superovulatory response to the number of transferable structures and embryos were the variables that were significantly affected, allowing to conclude that Simmental heifers had an optimal superovulatory response to the applied protocol.

\section{INTRODUCCIÓN}

La transferencia embrionaria se utiliza, a nivel mundial, en la reproducción de vacas para el mejoramiento de la calidad genética y para superar problemas de fertilidad, como los causados por estrés calórico (Hansen, 2007). Sin embargo, desde finales de los años ochenta la producción de embriones in vitro se ha utilizado satisfactoriamente. Por ende, la superovulación resulta una gran técnica para la obtención de embriones.

El éxito de un programa de transferencia de embriones se mide por el número de terneros que nacen vivos por hembra donante, en un determinado lapso de tiempo (Bo et al., 2006). Una de las

\footnotetext{
'Médico Veterinario. Investigador del IGBI y docente UNTRM. Correo electrónico: nmurga.fizab@untrm.edu.pe

${ }^{2}$ Médico Veterinario. Investigador del IGBI y docente UNTRM . Correo electrónico: jhony.gonzales@untrm.edu.pe

${ }^{3}$ Médico Veterinario. Investigador del IGBI y docente UNTRM. Correo electrónico: william.bardales@untrm.edu.pe
} 
limitantes de la transferencia de embriones es la variación existente en la respuesta superestimulante en bovinos. La sincronía de la ovulación es uno de los factores reportados como causa de pobre respuesta superestimulatoria. Se sabe también que una proporción importante de oocitos y embriones no son viables luego del tratamiento de superovulación y, aunque la célula espermática también contribuye, lo hace en menor proporción (Boerke et al., 2007). El origen del oocito determina el éxito del desarrollo embrionario temprano (Van Soom et al., 2007).

Entre los protocolos de superestimulación usados para sincronizar la emergencia de la onda folicular se emplea comúnmente el estradiol (E2) y los dispositivos de liberación de progesterona (P4) (Baruselli et al., 2006). En estos protocolos, el tratamiento con hormona folículo estimulante (FSH) inicia cuatro días después del tratamiento de E2 más P4 y está presente por varios días para sostener el crecimiento de los folículos, el cual es cumplido por la gonadotropina coriónica equina (eCG) (Bo et al., 2006). La continua actividad del FSH provee mediante señales a dos diferentes niveles, que controlan los cambios para el crecimiento folicular y alcanzar el desarrollo del oocito. La ovulación es controlada mediante el retiro del dispositivo de P4 por 24 o 36 h. después de la aplicación de prostaglandina PGF2 $\alpha$, y luego la administración de inductores de ovulación como la hormona liberadora de gonadotropina $(\mathrm{GnRH}$ o LH) en un tiempo fijo a partir de ese momento (Barros y Nogueira, 2001; Chesta et al., 2007).

Los trabajos mencionados anteriormente han sido llevados a cabo en diferentes razas de bovinos, no obstante, no se tiene muchos reportes en la raza Simmental. Por ello, el objetivo del presente trabajo fue evaluar la eficiencia de la respuesta superovulatoria de un protocolo en vaquillas de la raza Simmental en el núcleo genético de la UNTRM.

\section{MATERIAL Y MÉTODOS}

\section{Tipo de estudio}

Se realizó un estudio de tipo experimental y prospectivo.

\section{Sitio de estudio}

Se llevó a cabo en dos estaciones experimentales (E.E.) de la Universidad Nacional Toribio Rodríguez de Mendoza: E.E. Huambo ubicada en la provincia de Rodríguez de Mendoza y la E.E. Pomacochas en la provincia de Bongará, región Amazonas. La E.E. de Huambo se encuentra en una zona de bosque muy húmedo Premontano Tropical, a una altitud de $1967 \mathrm{~m} \mathrm{s.} \mathrm{n.} \mathrm{m.,} \mathrm{temperatura} \mathrm{prome-}$ dio de $17^{\circ} \mathrm{C}$, humedad relativa de $84 \%$ y precipitación anual de $1500 \mathrm{~mm}$. Por otro lado, la E.E. Pomacochas se localiza en una zona de bosque húmedo Montano Bajo Tropical, a una altitud de 2230 m s. n. m., temperatura promedio de $14{ }^{\circ} \mathrm{C}$ y precipitación anual de $3300 \mathrm{~mm}$ (IIAP y GRA, 2010).

\section{Unidades experimentales}

Se utilizaron como donadoras once vaquillas de la raza Simmental, cíclicas, con edades entre tres y cinco años, con una condición corporal de 3,5 y óptimas condiciones de reproducción. De las once donadoras: dos estuvieron en la E.E. Huambo y nueve en E.E. Pomacochas. En la E.E. de Huambo fueron manejados de manera semiintensiva, con tres horas de pastoreo en pasturas de Brachiaria por las mañanas y el resto del día de manera estabulada en corrales alimentadas con pasto de corte, king grass morado y verde, maralfalfa, ensilado de maíz y concentrado de mantenimiento. En la E.E. de Pomacochas, el manejo fue extensivo en pasturas de raigrás asociado con trébol y llevados a corrales en las noches, además se suministró ensilado de maíz, concentrado de mantenimiento, en los meses de escasez de pastos por abundantes precipitaciones (de enero a abril). Todos los animales del estudio tuvieron sal mineral y agua ad libitum.

Selección y superovulación de hembras donadoras La presente investigación consideró únicamente como donadoras a vaquillas, porque estas muestran mayor porcentaje de embriones viables que las vacas lactantes y no lactantes superovuladas (Peippo et al., 2009; Sartori et al., 2002).

Las donadoras recibieron un dispositivo intravaginal de Progesterona 1,38 g (CIDR ${ }^{\oplus}$, Pfizer) y $2 \mathrm{mg}$ de Benzoato de Estradiol (Estrovet ${ }^{\circledast}$, Montana) vía intramuscular (día 0), al cuarto día se inició el tratamiento de superestimulación ovárica con una dosis total de $240 \mathrm{mg}$ de FSH (Folltropin-V, Bioniche ${ }^{\circledR}$ ), administrada durante tres días consecutivos, en dosis decreciente, a intervalos de $12 \mathrm{~h}$. En la tarde del día seis, se inyectó vía intramuscular $25 \mathrm{mg}$ de PGF2 $\alpha$ (Lutalyse ${ }^{\circledR}$ ). En el día siete, se aplicó la segunda dosis de PGF2 $\alpha$ (en la mañana) y dos dosis de 200 UI de eCG (Folligon ${ }^{\circledR}$, Intervet), a intervalos de $12 \mathrm{~h}$. Por la mañana del día ocho, se aplicó GnRH (Acetato de Buserelina, Conceptal ${ }^{\circledR}$, Intervet) en dosis única de $3,5 \mathrm{ml}$, por la tarde (6:00 pm) se realizó la primera inseminación artificial (IA), que se repitió a las 12 horas con semen del mismo toro.

Las donadoras se superovularon en grupos de tres. Las vaquillas, que fueron sometidas al experimento más de una vez, tuvieron un período de 
descanso de al menos dos meses, para un máximo de nueve superovulaciones por vaquilla. De las once donadoras del experimento, cuatro recibieron entre una a tres superovulaciones; otras tres, de cuatro a seis y cuatro fueron superovuladas de siete a más veces.

\section{Recuperación, evaluación y clasificación de embriones}

La recuperación de embriones se realizó mediante un método no quirúrgico a los siete días después de la primera IA (día 15 del Protocolo, en la mañana). Previamente, se aplicó anestesia epidural a la donante, y luego se introdujo y se fijó un catéter armado a partir de una sonda Foley en la curvatura mayor de cada cuerno uterino. Esta sonda, a su vez, se conectó a un circuito cerrado de dos vías, a través de una de ellas, se introdujo $500 \mathrm{ml}$ de medio de lavado (BioLifeTM, Agtech), de manera interrumpida, en cantidades de 30 y 50 $\mathrm{ml}$; y a través de la otra vía, se recogió el medio con los embriones en un filtro (Zona TM Filter, Agtech), donde son retenidas las estructuras. Para la evaluación y clasificación de embriones se consideró el criterio de las normas de la Sociedad Internacional de Transferencia de embriones (IETS, 1998) y se utilizó un estereoscopio Nikon SM645, con $100 x$ de aumento.

\section{Respuesta a los tratamientos de superovulación} sucesivos

Para efectos de evaluar las respuestas a los tratamientos de superovulación sucesivos, se agruparon las respuestas de los animales superovulados entre una a tres veces, entre cuatro a seis veces y siete a más veces.

Respuesta superovulatoria de donadoras con una producción promedio menor a cinco $y$ mayor o igual a cinco embriones transferibles

Se agruparon a las vaquillas, de acuerdo con la producción promedio de embriones transferibles (mayor o igual a cinco y menor a cinco), durante los 58 lavados realizados, con la finalidad de comparar las respuestas superovulatorias en ambos grupos.

\section{Análisis estadístico}

Antes de realizar cada prueba para determinar diferencias entre los grupos respecto a las variables utilizadas, se realizó la prueba de Kolmogorov-Smirnov para comprobar la distribución normal entre los grupos de estudio. Para la determinación del número de estructuras recuperadas, estadio de desarrollo embrionario, número de embriones transferibles y degenerados del total de colectas, se realizó la prueba de hipótesis con intervalos de confianza al $95 \%$, y comparada con los datos reportados en la literatura sobre estas mismas variables.

La evaluación del efecto que ejerce el número de superovulaciones sobre la respuesta ovárica se realizó a través de un análisis de varianza (ANVA), se establecieron diferencias significativas por medio de las pruebas de Duncan y Tukey. La evaluación de la respuesta superovulatoria de las donadoras con una producción promedio mayor o igual, y menor a cinco embriones transferibles se realizó mediante la prueba de $\mathrm{T}$ de Student.

\section{RESULTADOS}

Los resultados de la tabla 1 muestran que el número de embriones transferibles de donadoras Simmental por colecta fue de $4,26 \pm 3,56$.

Tabla 1. Número de estructuras, embriones transferibles, degenerados y ovocitos no fertilizados de donadoras Simmental

\begin{tabular}{lc}
\hline \multicolumn{1}{c}{ Clasificación } & Datos de Donadoras * \\
\hline Número de animales & 11 \\
Número de Estructuras & $7,18 \pm 4,33$ \\
Embriones Transferibles & $4,26 \pm 3,56$ \\
Embriones Degenerados & $1,76 \pm 1,99$ \\
Ovocitos no Fertilizados (UFO) ${ }^{* *}$ & $1,16 \pm 2,31$ \\
\hline * Los resultados corresponden al promedio de un total de 58 \\
réplicas, en 11 donadoras. \\
** En la redacción los ovocitos no fertilizados serán citados \\
como UFO, que son las siglas del inglés Unfertilized Oocyte.
\end{tabular}

La tabla 2 muestra que los estadios de desarrollo embrionario predominantes fueron mórula compacta y blastocisto temprano con $80,16 \%$ y $12,55 \%$ respectivamente.

Tabla 2. Porcentajes de estadios embrionarios obtenidos de las donadoras Simmental

\begin{tabular}{lcc}
\hline $\begin{array}{c}\text { Estadio de desarrollo } \\
\text { embrionario }\end{array}$ & $\begin{array}{c}\text { Número de } \\
\text { embriones }\end{array}$ & $\begin{array}{c}\text { Porcentaje } \\
(\%)\end{array}$ \\
\hline Mórula compacta & 198 & 80,16 \\
Blastocisto temprano & 31 & 12,55 \\
Blastocisto & 14 & 5,67 \\
Blastocisto expandido & 4 & 1,62 \\
Totales & 247 & 100 \\
\hline
\end{tabular}

Con respecto al número de estructuras y embriones transferibles, la tabla 3 indica que existe diferencia significativa $(p<0,05)$ entre los tres grupos de donadoras y se observó que las donadoras con un número mayor e igual que siete superovulaciones presentan mejores resultados, además los embriones degenerados y ovocitos no fertilizados mostraron no ser significativos según el número de superovulaciones. 
Tabla 3. Efecto del número de tratamientos superovulatorios sucesivos sobre la respuesta superovulatoria en donadoras Simmental

\begin{tabular}{lccc}
\hline Clasificación & $\begin{array}{c}\text { Donadoras con 1-3 } \\
\text { Superovulaciones } \\
(\mathrm{n}=4)\end{array}$ & $\begin{array}{c}\text { Donadoras con 4-6 } \\
\text { Superovulaciones } \\
(\mathrm{n}=3)\end{array}$ & $\begin{array}{c}\text { Donadoras con } \geq 7 \\
\text { Superovulaciones }^{* * *} \\
(\mathrm{n}=4)\end{array}$ \\
\hline Número de Estructuras & $6,43 \pm 5,29^{\mathrm{ab}}$ & $4,94 \pm 3,49^{\mathrm{a}}$ & $8,34 \pm 3,94^{\mathrm{b}}$ \\
Embriones Transferibles & $3,43 \pm 5,26^{\mathrm{ab}}$ & $2,19 \pm 2,24^{\mathrm{a}}$ & $5,37 \pm 3,14^{\mathrm{b}}$ \\
Embriones Degenerados & $2,57 \pm 1,40^{\mathrm{a}}$ & $1,44 \pm 1,69^{\mathrm{a}}$ & $1,74 \pm 2,17^{\mathrm{a}}$ \\
Ovocitos no Fertilizados (UFO) & $0,43 \pm 0,73^{\mathrm{a}}$ & $1,31 \pm 2,36^{\mathrm{a}}$ & $1,23 \pm 2,46^{\mathrm{a}}$ \\
\hline
\end{tabular}

Letras diferentes en la misma fila indican diferencia estadística $(\mathrm{p}<0,05)$

\section{DISCUSIÓN}

El número promedio de estructuras recuperadas por colecta fue de 7,18 que es similar $(p>0,05)$ al encontrado por Martins et al. (2005) en vacas Nelore (7,4 estructuras) y al de Bo et al. (2006) en vacas y novillas Angus y Brangus, con un promedio de 9,1 estructuras recuperadas.

Respecto al número promedio de embriones transferibles recuperados por colecta en vacas Simmental se obtuvo 4,26 $\pm 3,56$, similar estadísticamente $(\mathrm{p}>0,05)$ a lo reportado por Bo et al. (2006) en vacas Angus y Brangus quienes obtuvieron 5,4 embriones transferibles por donadora. De igual manera Martins et al. (2005) y Baruselli et al. (2003) obtuvieron promedios similares de 6 y 6,5 embriones transferibles en vacas Nelore.

El número promedio de embriones degenerados obtenido en vacas Simmental fue de 1,76 \pm 1,99, similar $(\mathrm{p}>0,05)$ a lo reportado por Martins et al. (2005) en vacas Nelore, de 0,7 embriones degenerados. Sin embargo, Zanenga et al. (2003) comunicaron un promedio superior a 4,8 embriones degenerados por donadora en vacas Nelore.

La presencia de embriones degenerados y ovocitos no fertilizado es algo muy común luego de realizar la superovulación. Esto se debe a que la superovulación produce una disminución en más del $50 \%$ de la secreción endógena basal, de la frecuencia y amplitud de pulsos de la LH, tanto como un acortamiento del período de desarrollo folicular preovulatorio de 61 a $41 \mathrm{~h}$ en comparación a las vacas no estimuladas (Bevers y Dieleman, 1987). También, la superovulación induce cantidades anormales de esteroides en el suero comparado a niveles fisiológicos vistos en ciclos naturales. Todo lo anterior afecta adversamente la maduración celular de la granulosa y los ovocitos y compromete no solo la fertilización, sino también la viabilidad embrionaria (Hyttel et al., 1991). Con relación al estadio de desarrollo embrionario, el 80,16 \% obtenido perteneció al estadio mórula compacta, seguido de un 12,55 \% de blastocistos tempranos. Esto confirma que el protocolo utilizado, además de producir un promedio óptimo de embriones de buena calidad, permite sincronía en los eventos fisiológicos como el de la ovulación y fertilización de los ovocitos de las donadoras y, por tanto, en un mismo estadio embrionario. Al respecto, Palma (2008) reportó mayor cantidad de embriones en estadios de mórula y blastocisto temprano (37 \% de mórulas, $30 \%$ blastocistos tempranos y 32,9 \% mórulas, $26,4 \%$ blastocistos, respectivamente).

Los resultados relacionados a la producción de embriones transferibles, luego de varias superovulaciones sucesivas (2,19 vs. 5,37 para donadoras con 4-6 y con 7 o más superovulaciones, respectivamente), no solo no disminuyeron, sino que por el contrario aumentaron esta variable. Esto resultados coinciden a lo reportado por Hasler (2000), quien no encontró efecto negativo sobre esta variable en vacas Angus superovuladas en promedio 13,8 veces; y a lo reportado por Palma (2008), quien no halló variación en la producción de embriones transferibles a lo largo de tratamientos sucesivos.

\section{CONCLUSIONES}

La mayor producción de embriones transferibles $(7,58$ vs. 1,56$)$ se obtuvo a partir de vacas que alcanzaron una mejor respuesta superovulatoria (donadoras con producción $\geq 5$ embriones transferibles/superovulación). Esta mejor respuesta podría ser ocasionada por la variación en el número de folículos durante las ondas, ya que las vacas que presentan gran número de folículos durante las ondas foliculares responden mejor a la superovulación.

El protocolo de superovulación utilizado en vaquillas Simmental permitió una eficiente respuesta superovulatoria y producción embriones de buena calidad, similares a lo reportado en estudios actuales.

\section{REFERENCIAS BIBLIOGRÁFICAS}

Barros, C. M., \& Nogueira, M. F. G. (2001). Embryo transfer in Bosindicus cattle. Theriogenology, 56(9), 1483-1496.

Baruselli, P. S., de Sá Filho, M. F., Martins, C. M., Nasser, L. F., Nogueira, M. F., Barros, C. 
M., \& Bó, G. A. (2006). Superovulation and embryo transfer in Bos indicus cattle. Theriogenology, 65(1), 77-88.

Baruselli, P. S., Marques, M. O., Reis, E. L., Nasser, L. F. T., Silva, R. C. P., Menegatti, J. A., ... \& Santos, I. C. C. (2003). Adequação da dose de FSH (Folltropin-v) em protocolos de superovulação de vacas nelore (Bos taurus indicus) com inseminação artificial em tempo fixo (SOTF). Acta Scienciae Veterinariae, 31, 244-245.

Bevers, M. M., \& Dieleman, S. J. (1987). Superovulation of cows with PMSG: variation in plasma concentrations of progesterone, oestradiol, LH, cortisol, prolactin and PMSG and in number of preovulatory follicles. Animal Reproduction Science, 15(12), 37-52.

Bó, G. A., Baruselli, P. S., Chesta, P. M., \& Martins, C. M. (2006). The timing of ovulation and insemination schedules in superstimulated cattle. Theriogenology, 65(1), 89-101.

Boerke, A., Dieleman, S. J., \& Gadella, B. M. (2007). A possible role for sperm RNA in early embryo development. Theriogenology, 68, S147-S155.

Chesta, P., Tribulo, H., Baruselli, P., \& Bó, G. (2007). Effect of time of ovulation by gonadotrophin releasing hormone or pituitary luteinizing hormone on ova/embryo production in superovulated beef cows inseminated at a fixed time, 19:307.

Hansen, P. J. (2007). Exploitation of genetic and physiological determinants of embryonic resistance to elevated temperature to improve embryonic survival in dairy cattle during heat stress. Theriogenology, 68, S242-S249.

Hasler, J. F. (2000). Comparation between conventional in vivo and in vitro production of embryos in bovine embryo transfer programs. In Congreso de Reproducciòn Animal, Rosario, Argentina.

Hyttel, P., Callesen, H., Greve, T., \& Schmidt, M. (1991). Oocyte maturation and sperm transport in superovulated cattle. Theriogenology, 35(1), 91-108.

IIAP (Instituto de la Amazonía Peruana), GRA (Gobierno Regional Amazonas). (2010). Zonificación ecológica y económica del departamento de Amazonas. Lima, Perú. 196 p.

IETS (International Embryo Transfer Society). (1998). Manual of the international embryo transfer society. Stringfellow, D.A. Seidel, S.M. (Eds.). Illinois: International Embryos
Transfer Society, Inc.

Martins, C., Marques, M., Silva, R., Baruselli, P. (2005). Adecuación de un protocolo de superovulación con inseminación artificial a tiempo fijo en Bos Taurus. (Tesis de Maestría. Sao Paulo: Universidad de Sao Paulo).

Palma, G. (2008). Biotecnología de la reproducción. $2^{\text {a }}$. Ed. Mar de Plata: Reprobiotec.

Peippo, J., Vartia, K., Kananen-Anttila, K., Räty, M., Korhonen, K., Hurme, T., ... \& MäkiTanila, A. (2009). Embryo production from superovulated Holstein-Friesian dairy heifers and cows after insemination with frozen-thawed sex-sorted X spermatozoa or unsorted semen. Animal reproduction science, 111(1), 80-92.

Sartori, R., Sartor-Bergfelt, R., Mertens, S. A., Guenther, J. N., Parrish, J. J., \& Wiltbank, M. C. (2002). Fertilization and early embryonic development in heifers and lactating cows in summer and lactating and dry cows in winter. Journal of Dairy Science, 85(11), 2803-2812.

Van Soom, A., Vandaele, L., Goossens, K., de Kruif, A., \& Peelman, L. (2007). Gamete origin in relation to early embryo development. Theriogenology, 68, S131-S137.

Zanenga, C. A., Marques, M. O., Santos, I. C. C., Valentin, R., \& Baruselli, P. S. (2003). Comparação entre dois protocolos de superovulação com inseminação artificial em tempo fixo em vacas Nelore (Bos taurus indicus). Acta Sci Vet, 31(Suppl1), 626-7. 